\title{
Doxorubicin-Loaded Zein in Situ Gel for Interstitial Chemotherapy
}

\author{
Xiaoying Cao, ${ }^{a}$ Jianning Geng, ${ }^{a}$ Suwen $\mathrm{Su},{ }^{b}$ Linan Zhang, ${ }^{a}$ Qian Xu,${ }^{c}$ Li Zhang, ${ }^{d}$ Yinghua Xie, ${ }^{a}$ \\ Shaomei Wu, ${ }^{a}$ Yongjun Sun, ${ }^{a}$ and Zibin Gao*,a \\ ${ }^{a}$ Department of Pharmacy, Hebei University of Science and Technology; Yuhua East Road 70, Shijiazhuang 050018, \\ P. R. China: ${ }^{b}$ Department of Pharmacology, Hebei Medical University; Zhongshan East Road 361, Shijiazhuang \\ 050017, P.R. China: ' Department of CT, The Forth Hospital of Hebei Medical University; Jiankang Road 12, \\ Shijiazhuang 050011, P.R. China: and ${ }^{d}$ Department of Pharmaceutics, New Drug Research and Development Center, \\ North China Pharmaceutical Group Corporation; Heping East Road 388, Shijiazhuang 050015, P. R. China. \\ Received March 22, 2012; accepted July 15, 2012; advance publication released online August 1, 2012
}

A novel drug delivery system of doxorubicin (DOX)-loaded Zein in situ gel for interstitial chemotherapy was investigated in this study. The possible mechanisms of drug release were described according to morphological analysis by optical microscopy and scanning electronic microscope (SEM). In vitro and in vivo anti-tumor activity studies showed that DOX-loaded Zein in situ gel was superior to DOX solution. Local pharmacokinetics in tumor tissue was studied by quantitative analysis with confocal laser scanning microscopy (CLSM) combined with microdialysis technology. A pharmacokinetics mathematical model of DOX-loaded Zein in situ gel in tumors was then built.

Key words interstitial chemotherapy; in situ gel; laser scanning confocal microscopy; microdialysis; local pharmacokinetic mathematical model

Chemotherapy is one of the most important treatments for malignant tumors. However, malignant solid tumors, the highest incidence of multiple primary malignant tumors, has a special microenvironment that decreases the clinical efficacy of conventional chemotherapy. ${ }^{1)}$ Interstitial chemotherapy, first named by Brem, ${ }^{2)}$ presents an alternative to this conventional therapy. Recently, new formulations for interstitial chemotherapy have been developed, such as gliadel, ${ }^{3)}$ micro-chips, ${ }^{4)}$ nanoparticles, ${ }^{5)}$ polymeric wafers ${ }^{6)}$ and in situ gel. ${ }^{7)}$ In situ gel has become increasingly attractive as a carrier for drug delivery systems because it is injectable, is biodegradable, has simple drug loading and is convenient for dose adjustment. ${ }^{8-12)}$

Polymers used for in situ gels ${ }^{13-17)}$ are mainly synthesized. In this study, Zein was used as the base material of an in situ gel for interstitial chemotherapy. As a major storage protein, Zein is composed of about $45-50 \%$ of the total protein in corn. It was demonstrated to be a promising biomaterial with good biocompatibility for tissue engineering ${ }^{18-21)}$ and was also used for the preparation of microspheres for constant release of drugs. ${ }^{22)}$

To be a promising drug delivery system, it is important to explore the pharmacokinetics of drug-loaded Zein in situ gel. Regrettably, the study of local pharmacokinetics for drug delivery system (DDS) for interstitial chemotherapy has made little development due to the lack of feasible detecting methods for local drug concentrations within a tumor microenvironment. Confocal laser scanning microscopy (CLSM) is a type of high-resolution fluorescence microscopy that overcomes limitations of conventional wide field microscopy and facilitates the generation of high-resolution images from relatively thick sections of tissues. In addition, it has the capability of quantitative image analysis. ${ }^{23)}$ CLSM has mainly been used for image analysis, including cell recognition ${ }^{24)}$ and tissue imaging, ${ }^{25)}$ but quantitative CLSM for pharmacokinetics is rarely reported. In this study, we determined the local concentration of drug in a tumor through the quantitative image analysis of CLSM. This technology provides a method for the study of local pharmacokinetics in the antition for many hematological and solid tumors.

Doxorubicin (DOX) is a cytotoxic anthracycline that represents the first-line therapeutic option for many hematological and solid tumors. However, cardiotoxicity has limited its clinical application. ${ }^{26,27)}$ To reduce its toxicity and increase its selectivity, effective methods have been developed (e.g., co-delivery systems ${ }^{28)}$ and liposome $\operatorname{DDS}^{29)}$ ) for systemic administration to solid tumors. However, tumor localization and cardiotoxicity can still be improved. ${ }^{30,31)}$ Therefore, in this study, DOX-loaded Zein in situ gel was developed for interstitial chemotherapy. After intratumor injection, this system transforms from a liquid to a semisolid, which releases DOX locally to minimize toxicity and improve effectiveness.

\section{Experimental}

Materials DOX was obtained from Lingnan Pharmaceutical, Ltd., China. Zein was procured from Rixing Pharmaceutical Adjuvant Factory, China, and glycerol formal (GF) was obtained from Acros Organics, Belgium.

Formulation of the Delivery System Typically, Zein was dissolved in GF, ethanol and $\mathrm{H}_{2} \mathrm{O}(\mathrm{v}: \mathrm{v}: \mathrm{v}=30: 49: 21)$, which was left overnight to form a clear solution. For preparation of DOX-loaded Zein in situ gels, $40 \mathrm{mg}$ of DOX was added into the above solution and dissolved by stirring.

Morphology of Zein in Situ Gel In situ gels prepared were injected into phosphate buffered saline (PBS) to form gels. The gels were quickly frozen, sectioned in planes perpendicular to the gel surface and sequentially stained with hematoxylin and eosin (H\&E). A Motic DMBA400 Digital Biological Microscope (Xiamen, China) was used to analyze the gel samples.

In situ gels were injected into the release device containing PBS. The device was imbedded in a bathing constant temperature vibrator operating at $60 \mathrm{rpm}$ and $37^{\circ} \mathrm{C}$. After removing gel samples at the 1st and 48th hour after injection, the gel surface morphology was observed with scanning electronic microscope (SEM) (TM1000, Hitachi Company, Japan). Gel was then perpendicularly sectioned to the vertical axis and 
observed with SEM $12 \mathrm{~d}$ after injection.

In Vitro Release of DOX from in Situ Gels A $0.5 \mathrm{~mL}$ aliquot of DOX-loaded Zein in situ gel was added to $10 \mathrm{~mL}$ PBS (pH 7.4) containing $\mathrm{NaN}_{3}(0.05 \%, \mathrm{w} / \mathrm{v})$ and trypsin $(1.0 \%$, $\mathrm{w} / \mathrm{v}){ }^{32)}$ This system was shaken in a constant temperature oscillator at $50 \mathrm{rpm}$ and $37^{\circ} \mathrm{C}$. Five milliliter aliquots were then collected at predetermined time intervals and replaced with fresh buffer. The drug concentration was analyzed by HPLC.

Cell Culture Lewis lung carcer (LLC) cells, which were kindly provided by Hebei Medical University, were used in the experiment. LLC cells were cultured in Dulbecco's modified Eagle's medium (DMEM) supplemented with 10\% fetal bovine serum (FBS, PAA), $10 \mathrm{~mL} N$-(2-hydroxyethyl)piperazine- $N^{\prime}$-2-ethanesulfonic acid (HEPES), and $50 \mu \mathrm{g} / \mathrm{mL}$ penicillin and streptomycin. All other tissue culture reagents were obtained from Gibco (Grand Island, NY, U.S.A.). The cells were cultured at $37^{\circ} \mathrm{C}$ in a humidified atmosphere with 5\% $\mathrm{CO}_{2}$ (Thermo Scientific).

In Vitro Cytotoxicity Assay In vitro cytotoxicity was investigated using the WST- 1 assay. Briefly, $1.5 \times 10^{5}$ cells/well were plated in a 24-well plate (Jet, Canada) and incubated for $1 \mathrm{~d}$ in a humidified incubator. After $24 \mathrm{~h}$, the cells were added to physiological saline, blank Zein in situ gel, DOX solution or DOX-loaded Zein in situ gel (1 $\mu \mathrm{g}$ DOX/well). The 24-well plate was then maintained at the conditions mentioned above. After the first day, the cell culture medium was replaced with fresh medium without DOX. After 1, 2 and $4 \mathrm{~d}$, in vitro cytotoxicity for Lewis cells was compared using the WST-1 assay (Joint-Stock Company, Japan). Next, semisolid-gel was removed and $100 \mu \mathrm{L}$ of WST-1 reagent was added to each well. The plates were incubated in a humidified incubator for $4 \mathrm{~h}$, and then the samples were shaken for $1 \mathrm{~min}$. Finally, $100 \mu \mathrm{L}$ samples were removed from each well and transferred to a 96-well plate; absorbance was measured at $492 \mathrm{~nm}$ with a microplate reader (MK3, Thermo, U.S.A.).

In Vivo Anti-tumor Activity Experiments were performed with female C57BL/6J mice weighing $18 \pm 3 \mathrm{~g}$, age 6 to 8 weeks, raised at the SPF laboratory. The temperature of the environment was maintained at $25 \pm 2{ }^{\circ} \mathrm{C}$, and the animals were exposed to a 24-h circadian rhythm with free access to water and food. All experiments were performed in accordance with sound ethical principles.

Tumor models were established by heterotopic implantation with Lewis lung carcer cells. At adherence of $80 \%$, cultured cells were harvested with $0.25 \%$ trypsin and $1-5 \times 10^{6} / \mathrm{mL}$ was suspended in DMEM. The cell suspension $(200 \mu \mathrm{L})$ was then injected into one forefoot armpit, and the growth was regularly monitored.

In vivo experiments were conducted when the diameter of the tumor was approximately $1.0 \mathrm{~cm}$. Tumor volume were evaluated in intratumoral injection of physiological saline (control), DOX solution, blank Zein in situ gel, DOX-loaded Zein in situ gel and intravenous injection of DOX solution. The dosage of DOX in these experiments was $5 \mathrm{mg} / \mathrm{kg}$. Tumor volume was measured by a Somatom sensation 64 CT machine (Siemens, Germany) and determined with the software that came with the $\mathrm{CT}$ machine for automatic $3 \mathrm{D}$ reconstruction.

Establishing the Standard Curve of DOX Concentration and Fluorescence Intensity DOX solutions were prepared at concentrations of $2.4,9.6,12,24,48$ and $120 \mu \mathrm{g} / \mathrm{mL}$. A $10 \mu \mathrm{L}$

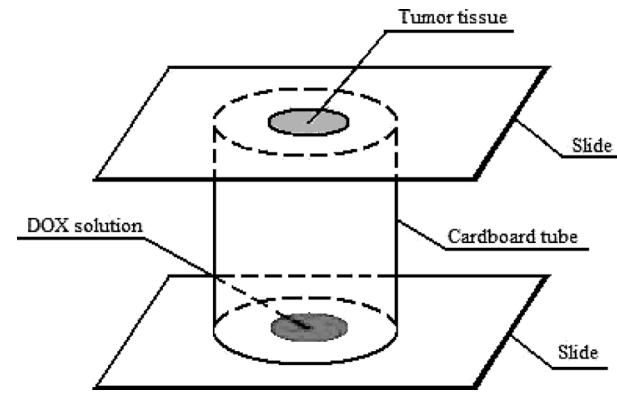

Fig. 1. Schematic Representation of the Apparatus Used to Evaluate the Influence of Tumor Tissue Thickness on DOX Fluorescence

aliquot of the DOX solutions was then dripped onto treated glass slides and put on the objective table of the CLSM. The argon ion laser was selected with an excitation wavelength of $488 \mathrm{~nm}$ and emission wavelengths of 580 to $610 \mathrm{~nm}$. The standard curve between DOX concentration and fluorescence intensity was thus built.

The accuracy was evaluated along with the recovery. The precision was represented by relative standard deviation (RSD) of DOX concentrations calculated with the standard curve. Low, medium and high normal saline DOX solutions were also measured by CLSM. The precision within a day was determined by testing DOX 6 times $(0,3,5,7,9,12 \mathrm{~h})$ in $1 \mathrm{~d}$ as well as between days by testing once a day for $5 \mathrm{~d}$. Then, RSD of the precision within and between days was calculated. The stability of the DOX normal saline solution was also observed. Each experiment was repeated three times.

Influence of tumor tissue thickness on the standard curve was also investigated. Briefly, blank tumor tissue sections were put on the object stage of the confocal laser scanning microscopy and scanned by the CLSM. A schematic of the experiment is presented in Fig. 1. As can be seen, a cardboard tube was between the glass slides. The DOX solution was in the underlying slide and the tumor tissue on the above slide. The laser beam was shined across the tissue to elicit the fluorescence of DOX.

Bound Fraction of DOX in Tumor Tissue The total DOX in the tumor was composed of the bound DOX and the free DOX. The free drug concentration was measured by microdialysis, and the total DOX concentration was measured by CLSM. The bound fraction of DOX in the tumor tissue could then be calculated.

Microdialysis sampling was used to measure the local free DOX in the tumor. The principle of the microdialysis probe was described in our previous work. ${ }^{33)}$ Briefly, the probe was perfused with a series of DOX solutions ranging from 2 to $64 \mu \mathrm{g} / \mathrm{mL}$, and its dialysis membrane was immersed into 20 $\mathrm{mL}$ saline containing $9.8 \mu \mathrm{g} / \mathrm{mL}$ DOX. After equilibration for $30 \mathrm{~min}$, the dialysate was collected and measured using HPLC to determine recovery and delivery in vitro.

A retrodialysis technique was then used for the assessment of microdialysis in vivo recovery. For this experiment, tumor-bearing C57 mice were anesthetized with 1\% pentobarbital $(45 \mathrm{mg} / \mathrm{kg})$. The probe was inserted into the tumor, and the perfusion solutions were perfused through the probe at a constant flow rate $(2 \mu \mathrm{L} / \mathrm{min})$. After allowing for a $2 \mathrm{~h}$ post-surgery stabilization period, the DOX concentration of perfusate $\left(C_{\mathrm{p}}\right)$ and dialysate $\left(C_{\mathrm{d}}\right)$ were determined using 
HPLC. Linearity between $C_{\mathrm{p}}$ and $\left(C_{\mathrm{d}}-C_{\mathrm{p}}\right)$ was established, and the slope of the line corresponded to the recovery $(R)$. Next, $0.2 \mathrm{~mL}$ of a $20 \mu \mathrm{g} / \mathrm{mL}$ DOX saline solution was injected in the tumors, and the probe was implanted in the tumor immediately. The probe was perfused with normal salt solution, and the free DOX concentration $\left(C_{\mathrm{f}}\right)$ in the tumor was calculated according to the following formula: $C_{\mathrm{f}}=\left(C_{\mathrm{d}}-C_{\mathrm{p}}\right) / R+C_{\mathrm{p}}$.

Total DOX in the tumor was determined by CLSM. Each tumor was injected with $0.2 \mathrm{~mL}$ of a $20 \mu \mathrm{g} / \mathrm{mL}$ DOX saline solution. Then, the mice were killed by the broken neck method at 65,85 , and $105 \mathrm{~min}$ post injection. The tumor was slivered along the injection direction, and the sections were frozen. The frozen sections were then scanned immediately by CLSM. The total drug concentration was calculated with the standard curve.

Pharmacokinetic Mathematical Model Once in situ gel was injected, local DOX concentrations in tumor tissue were determined by the dynamic balance between diffusion and elimination. A mathematical model based on the partial differential equation was established according to a previously published theory ${ }^{34)}$.

$$
\frac{\partial C}{\partial t}=\frac{D}{r} \cdot \frac{\partial\left[r \partial C_{\mathrm{f}} / \partial r\right]}{\partial r}-\gamma C_{\mathrm{f}}
$$

In this formula, $C$ is the total concentration at one sampling point, $t$ is the sampling time, $r$ is the distance between the sampling points and implants, $D$ is the diffusion coefficient, $C_{\mathrm{f}}$ is the free drug concentration, and $\gamma$ is the elimination coefficient.

(A) Mathematical Model for Different DOX Concentrations with the Same Prescription: The formulation of DOX-loaded Zein in situ gel were prepared with GF:ethanol: $\mathrm{H}_{2} \mathrm{O}$ $(\mathrm{v}: \mathrm{v}: \mathrm{v})=30: 49: 21$ and drug loading rates of 10, 20, 40, 60, and $120 \mu \mathrm{g} / \mathrm{mL}$. Each prescription contained 20\% Zein.

The mice in each experimental group were injected with $20 \mu \mathrm{L}$ of in situ gel with different DOX concentrations, while the control group was injected with DOX saline solution. The mice were sacrificed by the broken neck method at $6 \mathrm{~h}, 12 \mathrm{~h}$, $1 \mathrm{~d}, 2 \mathrm{~d}, 3 \mathrm{~d}$ and $7 \mathrm{~d}$ post injection. The tumors were dissected and put into a $-80^{\circ} \mathrm{C}$ refrigerator immediately. Frozen sections were scanned with CLSM, and the total drug concentrations in the local tumor tissue were calculated with the standard curve. The distance between sampling points and the DOX-loaded Zein in situ gel was measured using CLSM. The free drug concentration in the local tumor tissue was calculated using the bound fraction of DOX measured above. The diffusion coefficient, $D$, and elimination coefficient, $\gamma$, were calculated using Matlab (R2008a) software.

(B) Mathematical Model for the Same DOX Concentration with Different Prescriptions: The solvent for all prescriptions was GF : ethanol: $\mathrm{H}_{2} \mathrm{O}(\mathrm{v}: \mathrm{v}: \mathrm{v})=30: 49: 21$ at a concentration of $10 \mu \mathrm{g} / \mathrm{mL}$. In this experiment, Prescription (A) contained $20 \%$ Zein (v/v) and Prescription (B) contained 15\% Zein. $D$ and $\gamma$ were again calculated using Matlab software.

Statistical Analysis Significance between the mean values was calculated using analysis of variance (ANOVA) one-way analysis. $p$-Values $<0.05$ were considered significant.

\section{Results and Discussion}

Morphology of Zein in Situ Gel From direct observation, the gel was found to be coated with a "skin" on the surface of

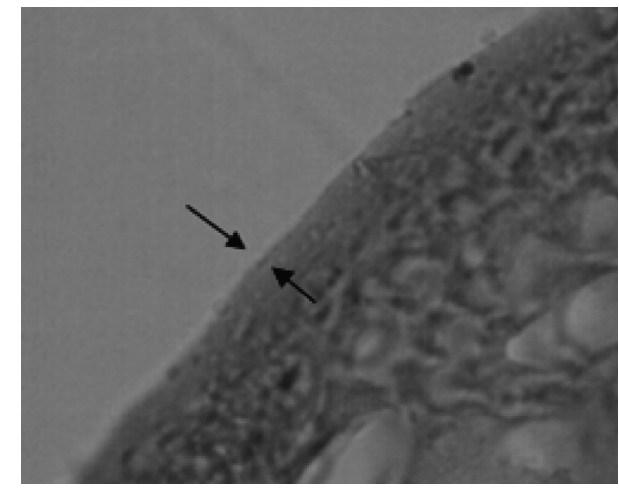

Fig. 2. The Diffusion Barrier (as Shown by the Arrow) of Zein in Situ Gel Stained by H\&E $(\times 1500)$

The diffusion barrier around the depot formed after contact with the aqueous buffer would be helpful to modulate the initial burst of water soluble drug.

porosity much lower than that of the interior (Fig. 2). In our previous work, ${ }^{32)}$ we demonstrated that the diffusion barrier around the depot formed after contact with the aqueous buffer would be helpful to modulate the initial burst of water soluble drug. The formation of this "skin" might be the result of rapid migration of Zein within the highly diffusive solvent toward the release buffer at the beginning of the gel forming process. This phenomenon then resulted in relatively more Zein being precipitated on the surface of the gel. With a decrease in solvent diffusion, the migration of Zein would not be remarkable, resulting in the interior of the gel becoming more porous than the exterior of the gel. ${ }^{32)}$

Figure 3A shows SEM images of the surface of the Zein in situ gel at the 1st hour. As can be seen, there were no obvious pores on the surface of the Zein in situ gel. However, at the 48th hour, the Zein in situ gel surface was eroded and many pores were observed on the surface (Fig. 3B). Figure 3C shows the interior of the Zein in situ gel at the 12th day. This reticular structure was interconnected and formed a diffusion barrier for DOX, but the porosity was much higher than that of the exterior (Fig. 2).

In Vitro Release of DOX-Loaded Zein in Situ Gel Figure 4 shows the in vitro release profile of DOX-loaded Zein in situ gels containing 15\%, 20\% and 25\% Zein with different solvents and drug loadings. Though there was an initial burst (45-65\%) in the first $24 \mathrm{~h}$, this delivery was still much lower than that of other in situ gel drug delivery systems for water soluble drugs. ${ }^{35)}$ With the increase in Zein concentration, the initial burst and the accumulative release percentage both decreased substantially. This finding might have been due to the increasing density and decreasing porosity of the Zein in situ gel. Interestingly, there was no significant difference between the accumulative release profiles for $0.2 \%$ and $0.4 \%$ DOX-loaded Zein in situ gel. Additionally, GF could slightly decrease this initial burst.

The solvent system for Zein in situ gel can be composed of both hydrophilic (e.g., $N$-methyl pyrrolidone (NMP) and ethanol) and hydrophobic (e.g., benzyl benzoate and sucrose acetate isobutyrate) components. ${ }^{11)}$ The hydrophilic solvent decreases the viscosity of the gel, which facilitates injection, resulting in a "skin" coating the interior part of the depot. The hydrophobic solvent slows water diffusion and therefore decreases protein hydrolysis. 

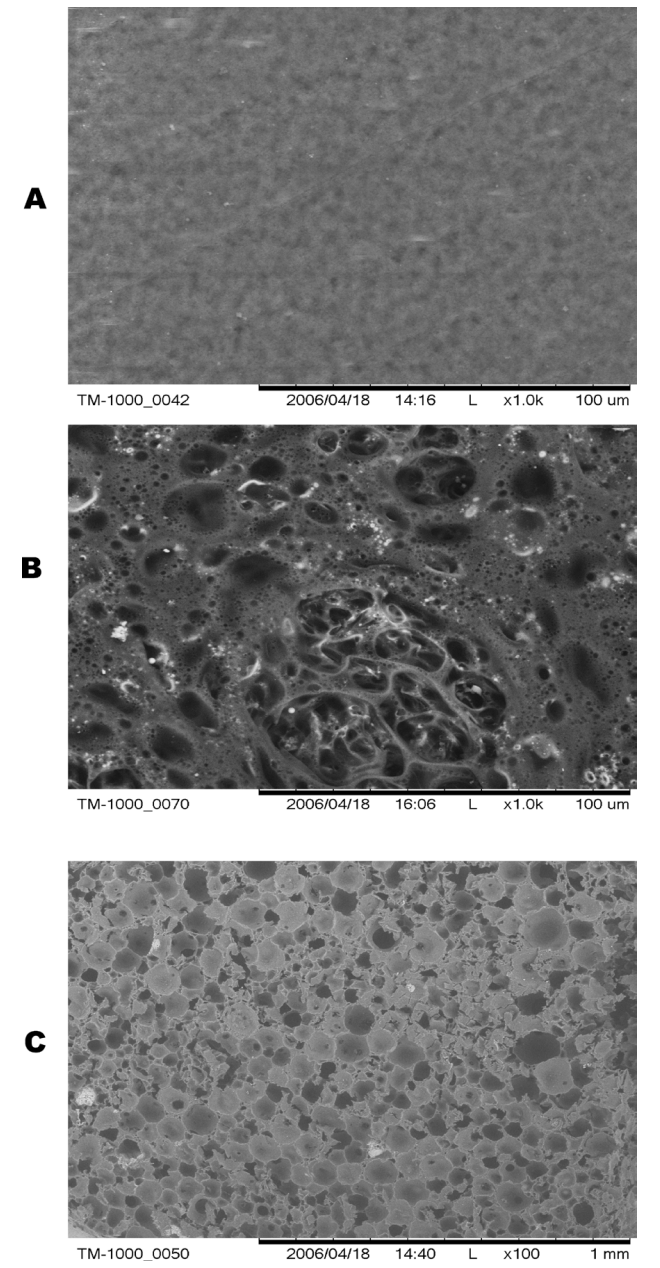

Fig. 3. SEM Images of the Surface of the in Situ Gel at the 1st Hour (A); 48th Hour (B)

There were no obvious pores on the surface of the Zein in situ gel at the 1st hour. However, at the 48th hour, the Zein in situ gel surface was eroded and many pores were observed on the surface (B). Figure 3C shows the interior of the Zein in situ gel at the 12th day. This reticular structure was interconnected and formed a diffusion barrier for DOX.

In Vitro Cytotoxicity Assay Table 1 shows the anti-proliferative activities against Lewis cells of the physiological saline group (control group), the blank Zein in situ gel group, the DOX solution group, and the DOX-loaded Zein in situ gel group. Proliferation was only slightly inhibited by addition of blank Zein in situ gel at the 1st day and had little inhibitory effect on cell proliferation $2 \mathrm{~d}$ later. Both the DOX solution and the DOX-loaded Zein in situ gel exerted significant inhibitory effects on cell proliferation at the 1 st $\mathrm{d}$, but the inhibition by DOX-loaded Zein in situ gel was higher at the 4th day. That might be resulted of the initial burst of DOX and the inhibitory effect of the solution of in situ gel as showed in blank Zein in situ gel group. The sustained release of DOX

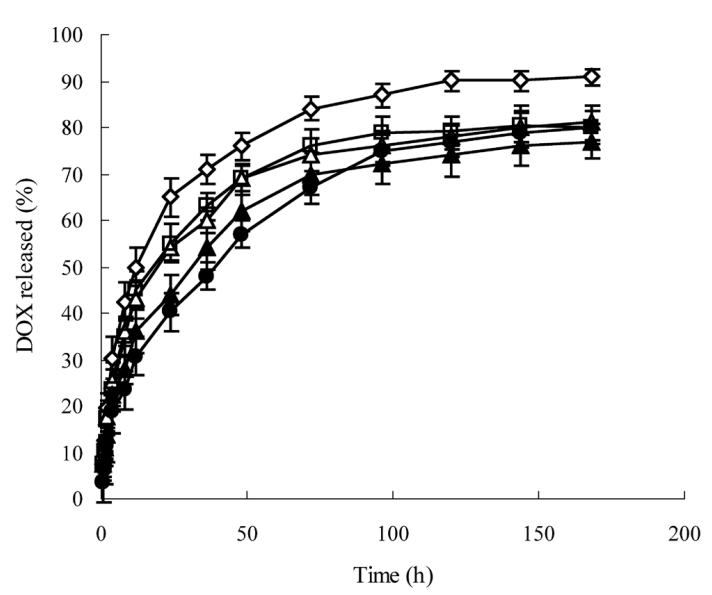

A

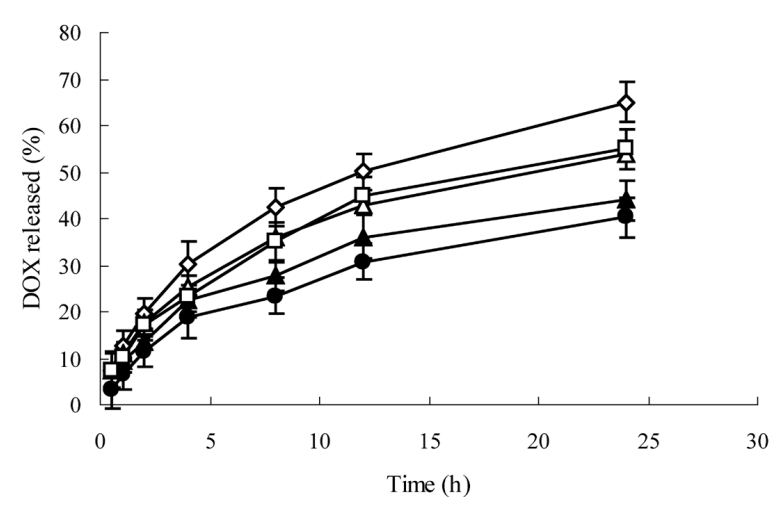

Fig. 4. (A) In Vitro Release of DOX from in Situ Forming Gel with Different Compositions; (B) In Vitro Release Profile within 24h to Show the Initial Burst of Different Compositions

$\diamond 15 \%$ Zein, $0.4 \%$ DOX, $\mathrm{H}_{2} \mathrm{O}$ : ethanol=3:7 (v:v); $\triangle 20 \%$ Zein, $0.4 \%$ DOX, $\mathrm{H}_{2} \mathrm{O}:$ ethanol=3:7 (v:v); $\boldsymbol{\Delta} 25 \%$ Zein, $0.4 \%$ DOX, $\mathrm{H}_{2} \mathrm{O}:$ ethanol=3:7 (v:v); $20 \%$ Zein, $0.4 \%$ DOX, GF: ethanol: $\mathrm{H}_{2} \mathrm{O}=30: 49: 21$ (v:v:v); $\square 20 \%$ Zein, $0.2 \%$ DOX, $\mathrm{H}_{2} \mathrm{O}$ : ethanol=3:7 (v:v).

from Zein in situ gel might be responsible for higher inhibition of cancer cell by DOX-loaded Zein in situ gel at the 4th day. These data further demonstrated that DOX-loaded Zein in situ gel has prolonged anti-tumor effects.

In Vivo Anti-tumor Activity The DOX-loaded Zein in situ gel with a Zein concentration of $20 \%(\mathrm{w} / \mathrm{w})$ and a solvent of $\mathrm{GF}$ : ethanol: $\mathrm{H}_{2} \mathrm{O}=30: 49: 21$ (v:v:v) was investigated because this composition was known to have the appropriate viscosity $(175 \pm 3 \mathrm{mPa} \cdot \mathrm{s}, n=3)$ and most desirable release profile for DOX. CT scanning 3D reconstructions were was applied to measure the tumor volume of vivi mice and to evaluate the in vivo anti-tumor effect of DOX-loaded Zein in situ gel (Figs. $5,6)$. The results indicated that DOX-loaded Zein in situ gel had an excellent anti-tumor effect. Because of the sustained release of DOX-loaded Zein in situ gel, the local DOX concentration in the solid tumor could be maintained above the

Table 1. The Anti-proliferative Activities against Lewis Cells of Each Drug Group (OD/492 nm)

\begin{tabular}{|c|c|c|c|c|}
\hline & Control & Blank Zein in situ gel & DOX solution $\left.^{a}\right)$ & DOX-loaded Zein in situ gel ${ }^{a}$ \\
\hline 1st day & $0.674 \pm 0.020$ & $0.637 \pm 0.065$ & $0.335 \pm 0.025$ & $0.329 \pm 0.021$ \\
\hline 2nd day & $0.813 \pm 0.012$ & $0.812 \pm 0.014$ & $0.277 \pm 0.075$ & $0.265 \pm 0.020$ \\
\hline 4th day & $0.884 \pm 0.010$ & $0.884 \pm 0.008$ & $0.324 \pm 0.018^{b)}$ & $0.217 \pm 0.040^{b)}$ \\
\hline
\end{tabular}

a) Significant difference compared with control group. $b$ ) Significant difference compared with each other $(p<0.05, n=5)$. 


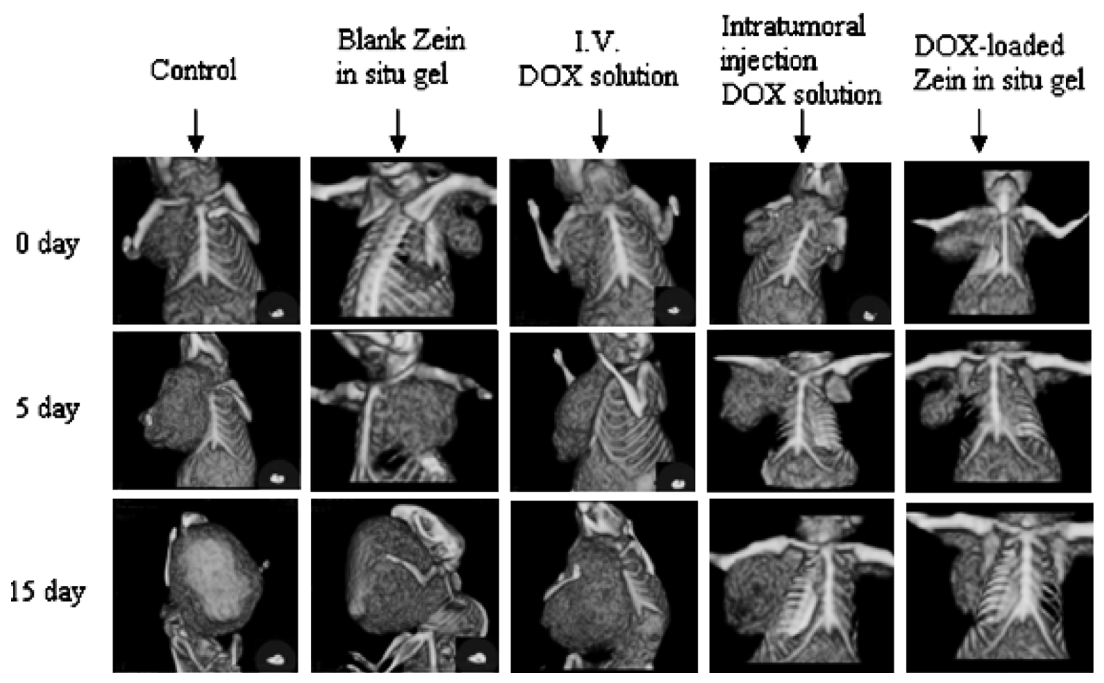

Fig. 5. Measurement of Tumor Volume of Mice by CT Scanning 3D Reconstructions

The results indicated that DOX-loaded Zein in situ gel had an excellent anti-tumor effect.

threshold concentration long term.

Standard Curve of DOX Concentration and Fluorescence Intensity The standard curve of DOX concentration versus fluorescence intensity was established by CLSM with a laser intensity of 706.7. The standard curve equation could be fitted by the hyperbolic equation $\ln F I=$ $-0.1125(\ln C)^{2}+1.241(\ln C)+2.065, \quad R^{2}=0.9985$. Doxorubicin concentrations from 2.4 to $120 \mu \mathrm{g} / \mathrm{mL}$ had a good linear response.

The accuracy, precision and stability of the drug solution were next investigated. Percent recovery was used to represented accuracy in this study and was found to be $97.33 \%$. The RSD of the within-day precision, the between-day precision and the stability of the drug solution were $0.7 \%, 1.28 \%$ and $1.21 \%$, respectively. In addition, the standard curve was inspected with different laser intensities. The results showed that different laser intensities affected the standard curve. Therefore, building of a separate standard curve was necessary for each experiment.

Blank tumor tissue sections were measured by CLSM and the results showed that blank tumor slices made as described above had no fluorescence in the excitation wavelength of DOX. The influence of tumor tissue thicknesses to standard curves was also measured. The results revealed that blank tumor slices of thickness from 20 to $40 \mu \mathrm{m}$ had no interference on the standard curve and did not affect the detection of DOX. However, when the thickness was above $50 \mu \mathrm{m}$, the interference became a problem for lower DOX concentrations. Therefore, $40 \mu \mathrm{m}$ was set as the maximum thickness of tumor tissue sections.

Bound Fraction of DOX in Tumor Tissue According to the definition for recovery, ${ }^{33)}$ the following equation was obtained: $C_{\mathrm{d}}-C_{\mathrm{p}}=-0.356 C_{\mathrm{p}}+3.2323\left(R^{2}=0.9994\right)$. The calculated in vitro recovery was then $35.6 \%(n=5$, S.D. $=2.3 \%)$. Because the recovery and delivery were almost identical, using delivery to predict recovery was reasonable and provided an experimental proof for in vivo measurement of recovery by retrodialysis. ${ }^{33)}$

To ascertain the in vivo concentration of free DOX, it was necessary to determine the recovery of the dialysis probe. The

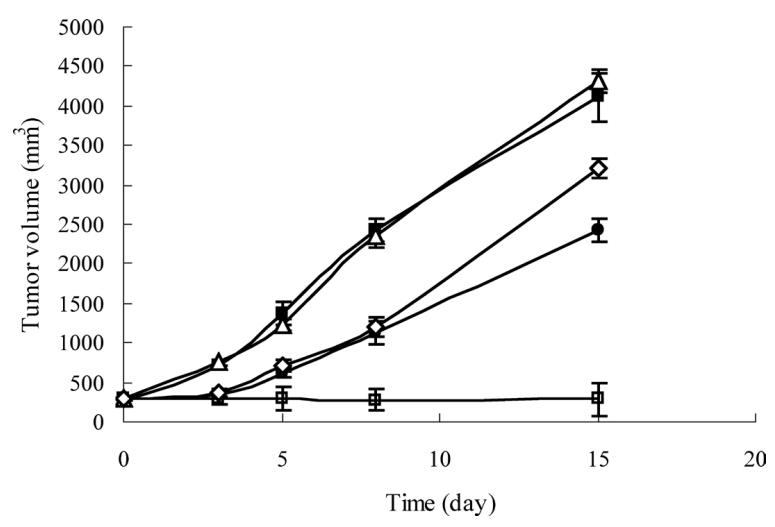

Fig. 6. Inhibitory Tumor Curve after Administration

Control (ष); Blank Zein in situ gel $(\triangle)$; i.v. DOX solution $(\diamond)$; Intratumoral injection DOX solution $(\bullet)$ and DOX-loaded Zein in situ gel $(\square)$ groups. Error bars represent standard deviations $(n=6)$.

following equation was obtained: $C_{\mathrm{d}}-C_{\mathrm{p}}=-0.3013 C_{\mathrm{p}}-0.104$ $\left(R^{2}=0.9989\right)$. The in vivo recovery calculated for DOX was $30.13 \%(n=5$, S.D. $=3.3 \%)$, which could then be used to determine the free DOX concentration in the tumor.

Table 2 shows that the DOX bound fraction in tumor tissue was $98.4 \%$. Therefore, the free drug concentration in the local tumor was calculated from the total concentration and the bound fraction of DOX in tumor tissue.

Mathematical Model After the DOX solution was injected into the tumor, the drug diffused along the decreasing direction of the drug concentration. Additionally, after DOX-loaded Zein in situ gel was injected into tumors, a complicated diffusion and elimination process was observed (Fig. 7). With increasing DOX concentration, the value of D increased gradually, changing from negative to positive (Table 3). In the mathematical models, a negative number for $D$ indicated the direction of drug diffusion was opposite to the increasing direction of the drug concentration; a positive number for $D$ indicated the direction of drug diffusion was the same to the increasing direction of the drug concentration. Because the stratum compactum of the in situ gel surface was built after the initial burst of in situ gel, drug release 
Table 2. Bound Fraction of DOX in Tumor Tissue $(n=5)$

\begin{tabular}{cccc}
\hline \hline Time $(\mathrm{min})$ & $\begin{array}{c}\text { Concentration } \\
(\mu \mathrm{g} / \mathrm{mL})\end{array}$ & $\begin{array}{c}\text { Free concentration } \\
(\mu \mathrm{g} / \mathrm{mL})\end{array}$ & Bound fraction \\
\hline 65 & 86.40 & 0.682 & 0.992 \\
85 & 94.91 & 0.603 & 0.991 \\
105 & 17.99 & 0.580 & 0.968 \\
\hline
\end{tabular}

became a sustained process (Fig. 8). Therefore, the local drug concentration in tumor tissues between the initial burst and the sustained release of in situ gel could be determined by the diffusion in these two directions and by the elimination. Moreover, with increasing drug concentration, tissue necrosis became more serious, which might have been the reason why $\gamma$ decreased when the DOX concentration of in situ gel was more than $60 \mu \mathrm{g} / \mathrm{mL}$. Therefore, further study is needed to determine whether $60 \mu \mathrm{g} / \mathrm{mL}$ is the threshold concentration for administration. Considering these observations, the local drug concentration in tumor tissue could remain higher than the threshold concentration for a longer period. Additionally, DOX-loaded Zein in situ gel had better therapeutic effect than DOX saline solution.

When concentration of Zein was reduced from 20 to $15 \%$, D decreased from -87.72 to $-386.78 \mu \mathrm{m}^{2} / \mathrm{h}$ (Table 4 ), possibly due to the weakening of the delayed release effect of the Zein in situ gel.

The Zein in situ gel designed for intratumoral injection described here was suitable for use as a sustained-release form of DOX and enhanced the anti-tumor effect of DOX in interstitial chemotherapy. Furthermore, by means of a pharmacokinetic mathematical model, we could predict further knowledge
Table 3. Values of $D$ and $\gamma$ for Different DOX Concentrations of in Situ Gel

\begin{tabular}{ccc}
\hline \hline$C_{\text {DOX }}(\mu \mathrm{g} / \mathrm{mL})$ & $D\left(\mu \mathrm{m}^{2} / \mathrm{h}\right)$ & $\gamma(1 / \mathrm{h})$ \\
\hline 10 & -87.72 & 2.33 \\
20 & -60.50 & 2.52 \\
40 & 14.73 & 3.33 \\
60 & 16.61 & 1.15 \\
120 & 226.27 & 1.10 \\
\hline
\end{tabular}

Table 4. Values of $D$ and $\gamma$ for Different in Situ Gels ${ }^{a)}$

\begin{tabular}{ccc}
\hline \hline Formulations & $D\left(\mu \mathrm{m}^{2} / \mathrm{h}\right)$ & $\gamma(1 / \mathrm{h})$ \\
\hline (A) & -87.72 & 2.33 \\
(B) & -386.78 & 3.46 \\
Control group & -1515.79 & 3.91 \\
\hline
\end{tabular}

a) The solvent for all prescriptions was $\mathrm{H}_{2} \mathrm{O}$ : ethanol: GF (v:v:v)=21:49:30 and the DOX concentration was $10 \mu \mathrm{g} / \mathrm{mL}$. Prescription (A): $20 \%$ Zein (v/v); Prescription (B): $15 \%$ Zein; Control group: DOX saline solution.

of the mechanism of release and action. All these data would provide the basis for individualized interstitial chemotherapy of DOX-loaded Zein in situ gel in the future.

Acknowledgements This study was supported by the Natural Science Foundation of China (NSFC, 30801444), the Natural Science Foundation of Hebei Province (C2009000693, H2012208020) and the Hebei University of Science and Technology Discipline Construction Office. We thank the Hebei Province Key Laboratory of Molecular Chemistry for Drug and the Hebei Provincial Tumor Hospital for help.

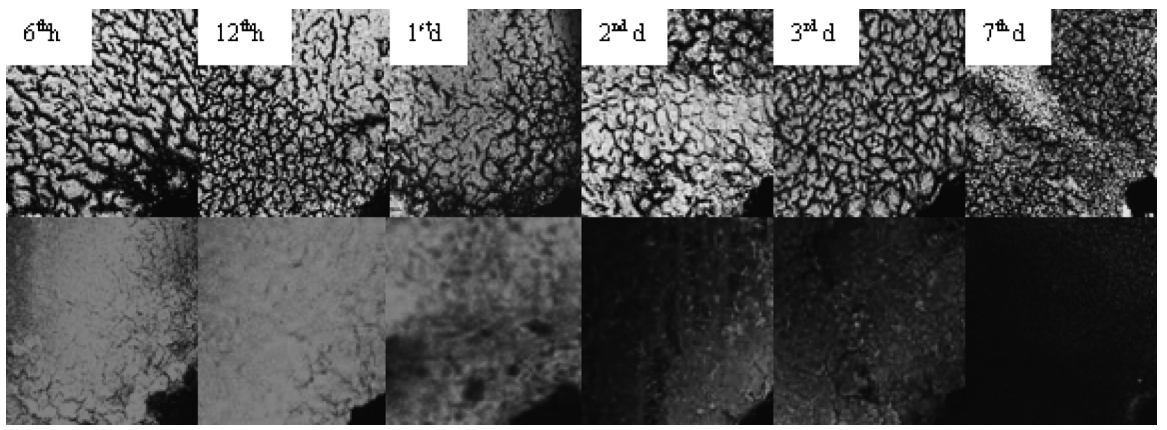

Fig. 7. Release of DOX-Loaded Zein in Situ Gel of $60 \mu \mathrm{g} / \mathrm{mL}$ (Upper Panel: Light Micrograph; Lower Panel: Imaging by CLSM)

After the injection of DOX-loaded Zein in situ gel into tumors, a complicated diffusion and elimination process was observed.

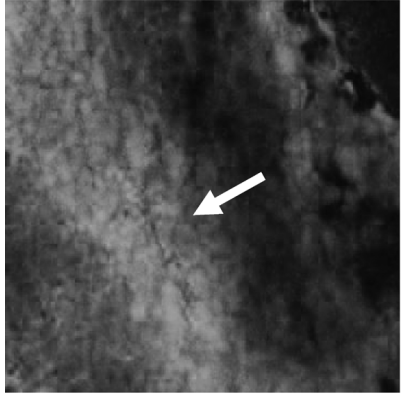

$\mathbf{A}$

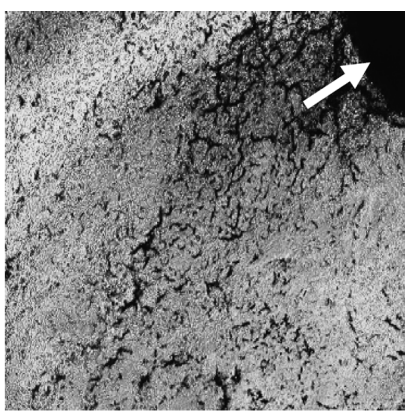

B

Fig. 8. Release of DOX-Loaded Zein in Situ Gel $(120 \mu \mathrm{g} / \mathrm{mL}$, 6th Hour)

(A) Imaging by CLSM (the initial burst shown by the arrow; (B) Light micrograph (in situ gel, shown by the arrow, was removed during sectioning). 


\section{References}

1) McCarron P. A., Woolfson A. D., Keating S. M., J. Pharm. Pharmacol., 52, 1451-1459 (2000).

2) Brem H., Mahaley M. S. Jr., Vick N. A., Black K. L., Schold S. C. Jr., Burger P. C., Friedman A. H., Ciric I. S., Eller T. W., Cozzens J. W., Kenealy J. N., J. Neurosurg., 74, 441-446 (1991).

3) Dörner L., Ulmer S., Rohr A., Mehdorn H. M., Nabavi A., J. Clin. Neurosci., 18, 347-351 (2011).

4) Kim G. Y., Tyler B. M., Tupper M. M., Karp J. M., Langer R. S., Brem H., Cima M. J., J. Controlled Release, 123, 172-178 (2007).

5) Roger M., Clavreul A., Venier-Julienne M. C., Passirani C., Montero-Menei C., Menei P., Biomaterials, 32, 2106-2116 (2011).

6) Arifin D. Y., Lee K. Y., Wang C. H., J. Controlled Release, 137, 203-210 (2009).

7) Liu W., MacKay J. A., Dreher M. R., Chen M. N., McDaniel J. R., Simnick A. J., Callahan D. J., Zalutsky M. R., Chilkoti A., J. Controlled Release, 144, 2-9 (2010).

8) Won Y. W., Kim J. K., Cha M. J., Hwang K. C., Choi D., Kim Y. H., J. Controlled Release, 144, 181-189 (2010).

9) Lee J. S., Zhou W., Meng F. H., Zhang D. W., Otto C., Feijen J., J. Controlled Release, 146, 400-408 (2010).

10) Censi R., Fieten P. J., Di Martino P., Hennink W. E., Vermonden T., J. Controlled Release, 148, e28-e29 (2010).

11) Jin R., Dijkstra P. J., Feijen J., J. Controlled Release, 148, e41-e43 (2010).

12) Park M. R., Chun C., Ahn S. W., Ki M. H., Cho C. S., Song S. C., J. Controlled Release, 147, 359-367 (2010).

13) Al-Abd A. M., Hong K. Y., Song S. C., Kuh H. J., J. Controlled Release, 142, 101-107 (2010).

14) Badi N., Lutz J. F., J. Controlled Release, 142, 224-229 (2009).

15) Anumolu S. S., Menjoge A. R., Deshmukh M., Gerecke D., Stein S., Laskin J., Sinko P. J., Biomaterials, 32, 1204-1217 (2011).

16) Jeong Y., Joo M. K., Bahk K. H., Choi Y. Y., Kim H. T., Kim W. K., Lee H. J., Sohn Y. S., Jeong B., J. Controlled Release, 142, 25-30 (2009).

17) Qi H. Y., Li L., Huang C. Y., Li W. M., Wu C. J., Chem. Pharm. Bull., 54, 1500-1507 (2006).
18) Tu J. W., Wang H. J., Li H. W., Dai K. R., Wang J. Y., Zhang X. L., Biomaterials, 30, 4369-4376 (2009).

19) Wang H. J., Gong S. J., Lin Z. X., Fu J. X., Xue S. T., Huang J. C., Wang J. Y., Biomaterials, 28, 3952-3964 (2007).

20) Gong S., Wang H., Sun Q., Xue S. T., Wang J. Y., Biomaterials, 27, 3793-3799 (2006).

21) Dong J., Sun Q., Wang J. Y., Biomaterials, 25, 4691-4697 (2004).

22) Liu X., Sun Q., Wang H., Zhang L., Wang J. Y., Biomaterials, 26, 109-115 (2005).

23) Jones C. W., Smolinski D., Keogh A., Kirk T. B., Zheng M. H., Prog. Histochem. Cytochem., 40, 1-71 (2005).

24) Li X., Zhou H. Y., Yang L., Du G. Q., Pai-Panandiker A. S., Huang X. F., Yan B., Biomaterials, 32, 2540-2545 (2011).

25) Kluza E., Yeo S. Y., Schmid S., van der Schaft D. W., Boekhoven R. W., Schiffelers R. M., Storm G., Strijkers G. J., Nicolay K., J. Controlled Release, 151, 10-17 (2011).

26) Kopecka J., Campia I., Olivero P., Pescarmona G., Ghigo D., Bosia A., Riganti C., J. Controlled Release, 149, 196-205 (2011).

27) Cai S., Thati S., Bagby T. R., Diab H. M., Davies N. M., Cohen M. S., Forrest M. L., J. Controlled Release, 146, 212-218 (2011).

28) Han L., Huang R. Q., Li J. F., Liu S. H., Huang S. X., Jiang C., Biomaterials, 32, 1242-1252 (2011).

29) Al-Abd A. M., Hong K. Y., Song S. C., Kuh H. J., J. Controlled Release, 142, 101-107 (2010).

30) Kim R. J., Peterson G., Kulp B., Zanotti K. M., Markman M., Gynecol. Oncol., 97, 374-378 (2005).

31) He B., Zeng J., Nie Y., Ji L., Wang R., Li Y., Wu Y., Li L., Wang G., Luo X. L., Zhang Z. R., Gu Z. W., Macromol. Biosci., 9, 1169-1175 (2009).

32) Gao Z. B., Ding P. T., Zhang L., Shi J., Yuan S. Q., Wei J., Chen D. W., Int. J. Pharm., 328, 57-64 (2007).

33) Gao Z. B., Ding P. T., Zhang L., Yuan Z. T., Shi J., Wei J., Chen D. W., Biomed. Chromatogr., 20, 1223-1228 (2006).

34) Gao J. M., Qian F., Szymanski-Exner A., Stowe N., Haaga J., J. Biomed. Mater. Res., 62, 308-314 (2002).

35) Okumu F. W., Dao N., Fielder P. J., Dybdal N., Brooks D., Sane S., Cleland J. L., Biomaterials, 23, 4353-4358 (2002). 